\title{
Investigation of Native Oxide Removing from HCPA ALD Grown GaN Thin Films Surface Utilizing HF Solutions
}

\author{
Petro Deminskyi, Ali Haider, Necmi Biyikli \\ UNAM \\ Bilkent University \\ Ankara, Turkey \\ p.deminskyi@gmail.com, \\ ali.haider@bilkent.edu.tr, \\ biyikli@unam.bilkent.edu.tr
}

\author{
Alexander Ovsianitsky, Alexander Tsymbalenko, Dmitry Kotov, \\ Vladyslav Matkivskyi, Nata Liakhova, Vladimir Osinsky \\ Research Institute of microdevices, \\ National Academy of Science of Ukraine \\ Kiev, Ukraine \\ alexander.persona@gmail.com, sashkoi1234@gmail.com, \\ kakbydima@gmail.com, matkivskyykp1@gmail.com, \\ lyahovann@gmail.com, osinsky@imd.org
}

\begin{abstract}
The paper consider oxygen contamination of HCPA ALD grown GaN films under an air conditioning and during different time duration. High resolution XPS analysis of HCPA ALD grown GaN films after diluted 1:10 HF(41\%) : $\mathrm{H}_{2} \mathrm{O}$ and undiluted HF (41\%) influence on oxygen impurities was investigated. Lesser oxygen impurities have been observed. Better resistivity to oxygen atoms of GaN thin films after diluted HF solution treatment was achieved compared to undiluted HF treatment and without treatment.
\end{abstract}

Keywords - GaN; HCPA ALD; oxygen; contamination; thin films.

\section{INTRODUCTION}

III-Nitrides (Al, In, Ga)N — are excellent semiconductor compounds, which are of significant research attention over the last 20-25 years. Their properties [1-5] such as wide energy bandgap, high electron saturation velocity, high breakdown fields, high operational temperatures, low thermal generation rates, radiation tolerance and biocompatibility led to many highly successful technologies in the electronic and photonic systems [6] (high power LED's [7], HEMT's [8, 9], multijunction solar cells $[10,11]$, future optical and memory devices, FETs, HEMTs and sensors [12], etc.).

Currently, atomic layer deposition (ALD) is one of the most promising low-temperature nanometric deposition techniques. It offers to obtain high conformal coatings even on very complex tridimensional surfaces, with a strict thickness tolerance. During an ALD cycle, only one molecular layer is deposited on the substrate surface, enabling the theoretical possibility to tailor the composition of the deposit up to molecular resolution [13]. The importance of ALD processes for high throughput and coating on complex device geometries is well recognized for these applications. Nevertheless this technique it still has to be improved for III-N compounds and their ternary solutions for obtaining better crystalline structures.

Moreover, there are still a lot of challenges that have to be solved for obtaining better crystalline structures and device quality layers: oxygen and carbon contamination [14], limited conformality [15], uniform doping [16], slowness of the process and others. Contamination of achieved films by carbon and oxygen is a challenging problem. This issue may exist because of insufficient purity of source gases and background contaminants in the vacuum. Oxygen contamination can also result from ex-situ exposure to air for polycrystalline materials. The existence of the native oxide layer on the air-exposed surface implied the possible creation of an uncontrolled dislocation formation that might significantly affect the next steps of a device fabrication process. It is one of the reasons of high densities of the threading dislocation (TDs) during GaN layer growth [17]. It can cause serious degradation processes in devices right up to complete failure of them.

Due to the necessity of transferring the samples from one tool to another, it is critical to protect atomic layer deposited films from atmospheric oxidation. A number of methods can help in solving the problem of contamination, among them are: HF treatment, annealing, capping layers [18], exposure to the H2 flux [19], etc. Additionally, this pollution can be addressed with gas purifiers, and by improving the vacuum system with the use of load locks, adequate attention to system leaks and having a good quality pumping system. Substituting alumina tubes for quartz can lead to some improvement [20].

Thus, our main goals is to understand: (1) how could we efficiently remove native oxide from the surface of III-nitride thin films grown using hollow cathode plasma assisted ALD; (2) how and for how long could we protect the film surfaces from oxidation for the next steps of device fabrication.

Previously reported results [21] provide evidence to believe that our further experiments will allow us to obtain III-nitride thin films grown using hollow cathode plasma assisted ALD that could be more stable to the outer environment after HFtreatment. Due to previous study of $\mathrm{HF}, \mathrm{HCl}$ (at room temperature) and $\mathrm{NH}_{4} \mathrm{OH}$ (at $50{ }^{\circ} \mathrm{C}$ ) treatments of MOCVD InAlN films [22], we used only HF solution in our experiments. 


\section{IEEE 36th International Conference on Electronics and Nanotechnology (ELNANO)}

\section{EXPERIMENTAL}

Gallium nitride film were deposited on Si (100) substrate at growth temperature $200{ }^{\circ} \mathrm{C}$ by using a Fiji LL Cambridge Nanotech ALD reactor equipped with remote inductively coupled RF-power source and base pressure 0.2 Torr. We used $\mathrm{GaEt} 3$ as a metal-organic precursor for Ga source. Ar is used as the carrier and plasma gas with 30 and $100 \mathrm{sccm}$ flow rates for $\mathrm{GaEt}_{3}$ and $\mathrm{N}_{2} / \mathrm{H}_{2}$ flows, respectively. A total of 1000 growth cycles were carried out at $300 \mathrm{~W}$ plasma power [23]. Film thickness and refractive index were estimated by using a visible range variable angle spectroscopic ellipsometry (SE) at three angles of incidence $(\psi(65,70,75), \Delta)$. The refractive index and thickness of GaN grown film were extracted from SE data by using the Cauchy dispersion function. Both undiluted (41\%) and diluted (1:10 of $41 \%)$ HF treatment of $\mathrm{GaN}$ films during $1 \mathrm{~min}$ at room temperature was used for our study. For better comparative results free of treatment GaN film was additionally used. For better understanding what is the effect of $\mathrm{HF}$ on $\mathrm{GaN}$ samples, we checked oxide contamination after $10 \mathrm{~min}, 20 \mathrm{~min}, 30 \mathrm{~min}, 1$ hour, 2 hours and 1 week of undiluted, diluted HF treatment and after the same periods of time for GaN film right after ALD growth without HF treatment. The elemental profiles and impurity incorporation of the GaN films after undiluted, diluted $\mathrm{HF}$ treatment and right after ALD growth were determined by Xray photoelectron spectroscopy (XPS) operating at monochromatized Al Ka wavelength.

\section{RESULTS AND DISCUSSION}

We explored the influence of HF treatment on the oxygen contamination of GaN films. The initial results for diluted HF treatment of GaN during 1 and 2 minutes still show presence of O1s after HF treatment that might be caused by oxygen diffusion inside few layers of $\mathrm{GaN}$ along the interface of air/GaN. Oxygen contamination on the surface was better for the sample which was treated with diluted HF during 1 minutes $(7,33 \%)$ comparing with sample after 2 minutes of HF treatment $(8,33 \%)$ (see Table 1$)$.

These contaminations differences between these two peaks might be caused by the different speed of GaN surface degradation during diluted and undiluted HF treatment that under a longer time make the surface after 2 min more nonuniform. Larger HF concentration might be a main reason of surface area increasing that lead to a faster oxidation under an air conditions. That is why for our further study we used HF treatment of $\mathrm{GaN}$ film during 1 minute.

The XPS O1s spectral intensity of GaN was reduced efficiently by HF-based solution at room temperature, compared with the sample without HF treatment (Table. 1). Because the oxide is removed mainly by HF, F1s group are also present.

The next aim of our study lies in characterization of surface oxidizing and oxygen contamination of GaN samples, after $10 \mathrm{~min}, 20 \mathrm{~min}, 30 \mathrm{~min}, 1$ hour, one week of HF treatment of $\mathrm{GaN}$ film surface.
TABLE I. ATOMIC \% OF GA3D, O1s, F1s, C1S AND N1S GROUPS AFTER 1 MIN HF TREATMENT OF GAN AND 10 MIN PRESENCE ON THE AIR

\begin{tabular}{|c|c|c|c|c|}
\hline № & Name & $\begin{array}{c}\text { Atomic \% } \\
\text { (without HF } \\
\text { treatment) }\end{array}$ & $\begin{array}{c}\text { Atomic \%o } \\
\text { (1 min HF } \\
\text { treatment) }\end{array}$ & $\begin{array}{c}\text { Atomic \% } \\
\text { (2 min HF } \\
\text { treatment) }\end{array}$ \\
\hline $\mathbf{1 .}$ & Ga3d & 30,63 & 24,91 & 24,77 \\
\hline $\mathbf{2 .}$ & O1s & 4,48 & 7,33 & 8,33 \\
\hline $\mathbf{3 .}$ & F1s & - & 4,1 & 3,87 \\
\hline $\mathbf{4 .}$ & C1s & - & 8,51 & 8,73 \\
\hline $\mathbf{5 .}$ & N1s & 63,97 & 55,14 & 54,31 \\
\hline
\end{tabular}

Figure 1 shows the difference in oxygen contamination for $\mathrm{GaN}$ film after different periods of time. We can make several observations on obtained results:

$-41 \% \mathrm{HF}, 1: 1041 \% \mathrm{HF}$ treat GaN film surface in a different ways. While $41 \% \mathrm{HF}$ removing the oxygen more effectively and open more ALD GaN surfaces, 1:10 41\% HF less active for $\mathrm{GaN}$ surface treatment; $\mathrm{GaN}$ film right after ALD growth process is more stable to the oxygen influence compared with 41\% HF, 1:10 41\% HF

- 10 minutes after $\mathrm{HF}$ treatment both GaN samples in 41\% HF and 1:10 41\% HF solutions have almost equal percentage of oxygen on the surface. Lower percentage of oxygen without HF is due to the XPS characterization of the sample immediately after ALD grown GaN that show more resistant stable to the oxidation processes under the air conditions;

- 30 minutes after HF treatment GaN sample in $41 \% \mathrm{HF}$ solution show lower oxygen contamination due to more effective oxygen removing from the sample and not long time duration after HF treatment. Oxygen contamination in the sample without HF treatment start to increase faster compared to two other samples;

- 1, 2 hours, 1 day, 1 week after HF treatment GaN sample in 1:10 41\% HF solution (compared with sample treated in undiluted HF solution) show better stability under air conditioning. After this point we see almost the same correspondences.

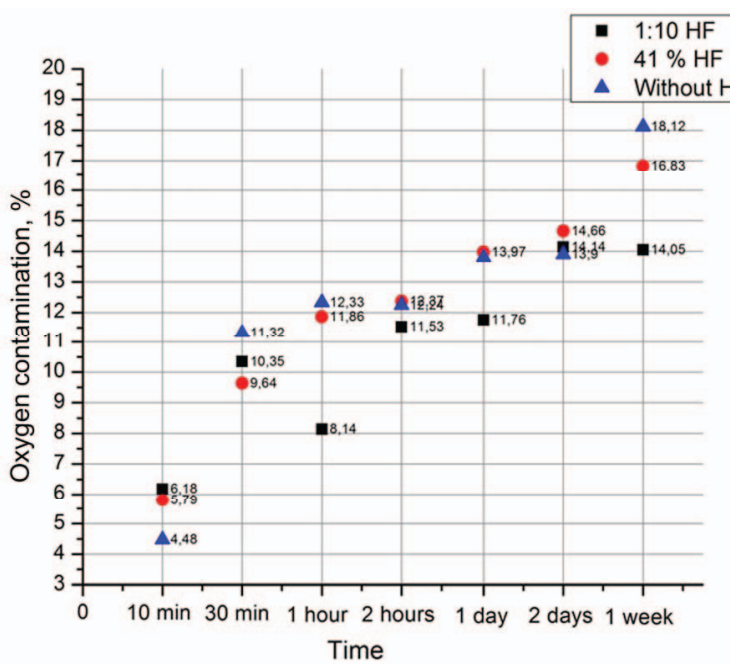

Fig. 1. Oxygen contamination of GaN after different time of air conditioning. 


\section{IEEE 36th International Conference on Electronics and Nanotechnology (ELNANO)}

As far as 1:10 HF solution seems the best choice for getting more resistant GaN top surface, we obtained high resolutions Ga3d, O1s and N1s peaks via XPS system and made parameters fitting of the surface of samples that were treated with 1:10 HF solution and their air conditioning during $10 \mathrm{~min}, 1$ hour and 1 week. For sample after $10 \mathrm{~min}$ under air conditioning we observe the main peak and first sub peak that reveals Ga3d signal on the surface. The second sub peak is $20.19 \mathrm{eV}$. This binding energy still related to Ga3d, nevertheless this slight shift to higher energy show initial surface modification with oxygen. In the literature $\mathrm{Ga}_{2} \mathrm{O}_{3}$ expected to be centered at $20.5 \mathrm{eV}$ or higher [24] that confirm our hypothesis. The XPS spectra show O1s peak on $531.07 \mathrm{eV}$ and coincide with literature [25].

The Ga3d and O1s XPS analysis of GaN sample after 1 hour of 1:10 HF treatment reveal a dominant subpeak at $532.48 \pm 0.1 \mathrm{eV}$, that corresponds to O-C peak (due to atmospheric contamination) that closely similar to a literature data $(532.6 \mathrm{eV})[26,27]$ and binding energy $\sim 19.68 \mathrm{eV}$ that represent narrow Ga3d signal. The presence of peak in a binding energy $20.07 \mathrm{eV}$ start to appear, that represent $\mathrm{Ga}-\mathrm{O}$ binding [28], while second sub peak $19.94 \mathrm{eV}$ increase in intensity. This sub peak might reveal to a second sub peak from samples with 10 min of air conditioning.

Ga3d XPS analysis of GaN sample after 1 week of 1:10 HF treatment reveal a dominant subpeak at $532.48 \pm 0.1 \mathrm{eV}$ and binding energies at $19.8 \mathrm{eV}$ and $20.07 \mathrm{eV}$, that represent $\mathrm{Ga}-\mathrm{O}$ bond and larger shifting to bigger energies, compared to 1 hour of 1:10 HF treatment of GaN film that related to $\mathrm{Ga}_{2} \mathrm{O}_{3}$. As we can see from Fig. 2a (After 1 week), Ga-O binding peak increase, while intensity of Ga3d decreasing.

High resolution XPS scan of N1s was collected to obtain oxidation state information about GaN film. The $\mathrm{N}$ 1s peak is commonly used for the identification of the nitrogen oxidation state. This is due to its high photoemission intensity [29]. The main peak that was obtained both after $10 \mathrm{~min} 397.18 \mathrm{eV}$ Ga-N binding [30] and 1 hour was $397.02 \mathrm{eV}$ that correspond to N-3 and represent nitride [31]. As seen in Fig. 2, another N1s peak is centered at $397.7 \mathrm{eV}$, which corresponds to the nitride oxidation state [32]. Peak at $395.7 \mathrm{eV}$ correspond to Ga Auger peaks [33].

Relative energy binding show slight shifting in intensity for $10 \mathrm{~min}$ and 1 hour (400 a.u.) while intensity comparison for samples after $10 \mathrm{~min}$ and 1 week under air conditions show decreasing in intensity up to 1150 a.u. For main peaks that related to N1s and Ga-N binding intensity decrease, while intensity of sub peaks increasing.

\section{CONCLUSION}

After detailed study of native oxide presence in GaN thin films after HF treatment and comparatively inconsiderable time under the air conditioning (10 min) we observed top GaN layers still contain $\sim 3-6 \%$ of oxide atoms depending on $\mathrm{HF}$ concentration and HF treatment time. Better resistivity was observed for GaN film without any HF treatment 10 min after HCPA ALD grown GaN. The resistivity of GaN film to oxygen incorporation decrease after enlargement of air conditioning time for samples (1) without and (2) with undiluted HF treatment., while for sample with utilization of diluted HF, GaN surface becomes more resistive in comparison to previous samples.
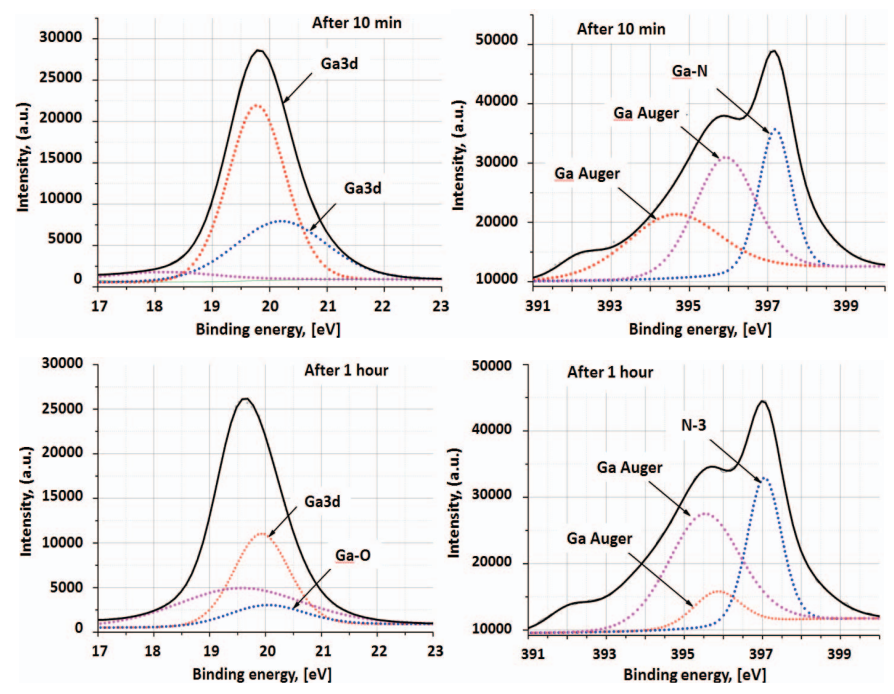

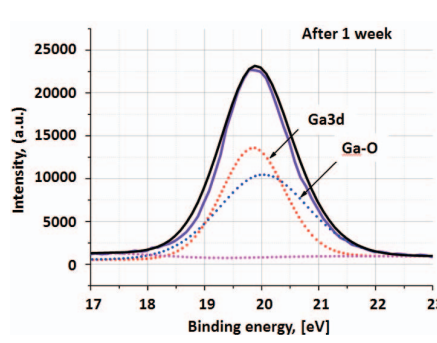

a

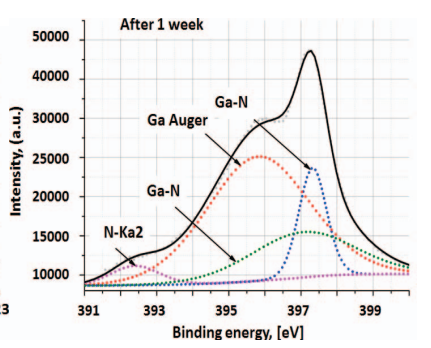

b
Fig. 2. High-resolution XPS spectra of (a) Ga3d and (b) N1s peaks of PEALD grown GaN films after different time of air conditioning.

GaN films after diluted 1:10 HF treatment of top surface show better resistivity to the air incorporation after $30 \mathrm{~min}, 1$ hour, 2 hours and 1 week under air influence that might be caused because of top surface oxygen saturation and better packaging of underneath GaN film.

HCPA ALD GaN grown films could be used for further technological steps where oxygen contamination on the surface in the range to $5-10 \%$ is not critical. Otherwise, other technological optimization steps for better resistivity or improved technique for native oxide removing from the top $\mathrm{GaN}$ are needed.

\section{ACKNOWLEDGMENT}

The This work was performed at UNAM under "2216 research fellowship program for international researchers" gratefully acknowledges the financial support from TUBITAK, UNAM, Institute of Material Science and Nanotechnology.

\section{REFERENCES}

[1] Simin $\mathrm{G}, \mathrm{Hu} \mathrm{X}$, Ilinskaya $\mathrm{N}$, et al. Large periphery high-power AlGaN/GaN metal-oxide-semiconductor heterostructure field effect transistors on $\mathrm{SiC}$ with oxide-bridging. IEEE Electron Device Lett, 2001, 22(2): 53

[2] Daumiller I, Theron D, Gaquiere C, et a1. Current instabilities in GaNbased devices. IEEE Electron Device Lett, 2001, 22(2): 62 


\section{IEEE 36th International Conference on Electronics and Nanotechnology (ELNANO)}

[3] Mishra U K, Zolper J C. Special issue on group III-V semiconductor electronics. IEEE Trans Electron Devices, 2001, 48: 405

[4] Wu Y F, Kapolnek D, Ibbetson J P, et al. Very-high power density AlGaN/GaN HEMTs. IEEE Trans Electron Devices, 2001, 48(3): 586

[5] N. Tsurumi, et al., "GaN Transistors for Power Switching and High Frequency Applications", Digest of Papers from the 2008 Compound Semiconductor and Circuits Symposium, pp. 1-5.

[6] Perspectives on future directions in III-N semiconductor research Charles R. Eddy Jr.1,a), Neeraj Nepal2,b), Jennifer K. Hite3 andMichael A. Mastro3

[7] V.I. Osinsky, P.V. Deminsky, etc. 8th All-Russian Conference "Gallium, aluminum and indium nitrides", St-Petersburg, Russia (2011)

[8] S. Tripathy, V. K. X. Lin, etc. Appl. Phys. Lett. 101 (8), 082110 (2012).

[9] K. Radhakrishnan, N. Dharmarasu, Appl. Phys. Lett. 97 (23), 232107 (2010).

[10] Jian-Wei Ho Journal of Crystal Growth 420, 64-73 (2015)

[11] Macho Microelectronics Journal 40, 427- 434 (2009)

[12] Elia Marin, Alex Lanzutti, Francesco Andreatta, Maria Lekka, Luis Guzman, Lorenzo Fedrizzi, Corrosion Reviews Volume 29, Issue 5-6 (Nov 2011) Atomic layer deposition: state-of-the-art and research/industrial perspectives

[13] Anjana Devi, Volume 257, Issues 23-24, December 2013, Pages 33323384, 'Old Chemistries' for new applications: Perspectives for development of precursors for MOCVD and ALD applications

[14] J. Musschoot, Q. Xie, D. Deduytsche, S. Van den Berghe, R.L. Van Meirhaeghe, C. Detavernier, Microelectronic Engineering 86 (2009) 7277 Atomic layer deposition of titanium nitride from TDMAT precursor

[15] Nicola Pinna,Mato Knez Atomic Layer Deposition of Nanostructured Materials p.67

[16] Lauri Niinistö, Atomic Layer Deposition of Rare Earth Oxides, Helsinki University of Technology p. 6

[17] E. A. Stach Appl. Phys. Lett. 79, 335 (2001)

[18] Chung-Mo Yang et al 2007 Jpn. J. Appl. Phys. 461981

[19] Interaction Between Plasma and Low-kappa Dielectric Materials, ProQuest, 2008, p.174
[20] N. M. Johnson, J. Walker, C. M. Doland, K. Winer and R. A. Street, Appl. Phys. Lett. 54 (1989) 1872.

[21] M. Akazawa, M. Chiba, and T. Nakano. CS MANTECH Conference, Denver, Colorado, USA (2014)

[22] Akazawa M., Chiba M., Nakano T. Process-dependent properties of InAlN surface and ALD-A12O3/InAlN interface CS MANTECH Conference, May 19th - 22nd, 2014, Denver, Colorado, USA

[23] M. Alevli, A. Haider, S. Kizir, S. A. Leghari, and N. Biyikli, J. Vac. Sci. Technol. A 34, 01A137 (2016).

[24] Giroire B., Marre S. Garcia A. Cardinal T., Aymonier C. Continuous supercritical route for quantum-confined $\mathrm{GaN}$ nanoparticles / The Royal Society of Chemistry, 2015

[25] Saygilıa H., Fuat Güzelb F. J. Chemical engineering research and design $10027-38,(2015)$

[26] NIST X-ray Photoelectron Spectroscopy Database, National Institute of Standards and Technology, http://srdata.nist.gov/XPS/

[27] P. Y. Jouan, M. C. Peignon, C. H. Cardinaud, G. Lemperiere, "Characterisation of TiN coatings and of the TiN/Si interface by x-ray photoelectron spectroscopy and Auger electron spectroscopy", Appl. Surf. Science, Vol.68, P.595 (1993).

[28] S. C. Ghosh, M. C. Biesinger, R. R. LaPierre, P. Kruse X-ray photoelectron spectroscopic study of the formation of catalytic gold nanoparticles on ultraviolet-ozone oxidized GaAs (100) substrates JOURNAL OF APPLIED PHYSICS 101, 1143222007

[29] Vasile M.J., Emerson A.D. Baiocchi F.A. "Growth and Characterization of Thin Titanium Nitride Films," J. Vac. Sci. Tech., A8, 99-105 (1990)

[30] Wei et al. Nanoscale Research Letters 2012, 7:562

[31] Kirkland W. Vogt and Paul A. Kohl Nitridation and CVD Reactions with Hydrazine / AIChE Journal, Vol 41, No 1, 1995

[32] Hedman J. Martensson N. Gallium nitride studied by electron spectroscopy. Phys. Scr., 22, 176 (1980)

[33] Kirste R., Rohrbaugh N., et al. Electronic Biosensors Based on IIINitride Semiconductors Annual Review of Analytical Chemistry Vol. 8: 149-169 (Volume publication date July 2015) 